\title{
Erwan Dianteill, La Samaritaine noire. Les Églises spirituelles noires américaines de la Nouvelle-Orléans
}

Paris, Éditions de l'École des hautes études en sciences sociales, Cahiers de l'Homme, 38, 2006, 262 p. (+ film documentaire DVD Roll with the Spirit)

\section{André Mary}

\section{OpenEdition}

\section{Édition électronique}

URL : http://journals.openedition.org/assr/3916

DOI : 10.4000/assr.3916

ISSN : $1777-5825$

Éditeur

Éditions de l'EHESS

\section{Édition imprimée}

Date de publication : 1 décembre 2006

Pagination : 115-283

ISBN : 2-7132-2124-2

ISSN : 0335-5985

Référence électronique

André Mary, «Erwan Dianteill, La Samaritaine noire. Les Églises spirituelles noires américaines de la Nouvelle-Orléans », Archives de sciences sociales des religions [En ligne], 136 | octobre - décembre 2006, document 136-35, mis en ligne le 12 février 2007, consulté le 21 septembre 2020. URL : http:// journals.openedition.org/assr/3916 ; DOI : https://doi.org/10.4000/assr.3916

Ce document a été généré automatiquement le 21 septembre 2020.

(c) Archives de sciences sociales des religions 


\section{Erwan Dianteill, La Samaritaine noire. Les Églises spirituelles noires américaines de la Nouvelle-Orléans}

Paris, Éditions de l'École des hautes études en sciences sociales, Cahiers de l'Homme, 38, 2006, 262 p. (+ film documentaire DVD Roll with the Spirit)

\section{André Mary}

1 Cet ouvrage ouvre une fenêtre sur une réalité religieuse des États-Unis plutôt ignorée ou méconnue du public francophone. Quand on parle aujourd'hui des Églises noires américaines, on pense aux Églises de la mouvance protestante et évangélique, plus précisément aux Églises baptistes ou pentecôtistes, dont les formes de prédication, et surtout les liturgies chantées et dansées inspirées du Gospel, font désormais le tour du monde. Les Églises «spirituelles », Spiritual Church, originaires de la Nouvelle-Orléans, sont moins connues et peuvent paraître, aux yeux des Églises mainstream, un peu folkloriques. Aujourd'hui en déclin, elles ont pourtant eu leur heure de gloire, et ont été largement étudiées par les chercheurs américains sur le plan sociologique et anthropologique (Baer \& Singer, 1992 ; Jacobs \& Kaslow, 1991). Un des premiers intérêts de ce travail original, issu d'une enquête très documentée, totalisant six mois de missions sur le terrain, est donc de faire connaître au public francophone ce que sont ces Églises - un réseau associatif de petites communautés segmentées -, et la place ambiguë qu'elles occupent dans l'espace religieux afro-américain. La manière dont elles ont été perçues par la société américaine et le trouble qu'elles suscitent dans les catégories de classement du champ d'étude de la sociologie des religions font partie de leur histoire (chap. 6).

2 L'opposition idéal-typique entre des religions «afro-latines» (afro-cubaines, haïtiennes) marquées par l'héritage de traits «africains » et des religions «noires » (chrétiennes ou islamiques), porteuses d'une conscience identitaire, culturelle ou politique, permet de souligner, d'emblée, l'ambivalence des Spiritual Churches dont la spiritualité doit beaucoup à une religiosité africaine inspirée du hoodoo (version 
louisianaise du vaudou) mais qui n'entend pas le reconnaitre et encore moins le revendiquer de manière protestataire. Les termes d'«africanité » et d'«africanisme " retenus par l'auteur (p. 28) pour désigner respectivement la simple présence de traits africains par opposition à leur revendication idéologique, risque par contre de prêter à confusion car l'africanité (comme l'ivoirité ou l'akanité), surtout dans la version initiatique à laquelle il est fait référence, renverrait plutôt à une identité revendiquée, et même "réafricanisée » (épurée de la sacralité catholique), tandis que, de son côté, l'africanisme (au moins dans le monde francophone) désigne un champ d'étude ou une tradition savante que les idéologies afro-centristes dénoncent comme un pur produit de l'euro-centrisme colonial. L'anthropologie « africaniste " peut retrouver ici tous les problèmes que posent, au regard des appellations indigènes autant que des typologies savantes, les caractéristiques hybrides des Églises dites indépendantes ou prophétiques africaines (type Églises aladura du Nigeria ou Églises zionistes d'Afrique du Sud), combinant la puissance de l'esprit des pentecôtismes, l'attachement à la sacralité rituelle catholique, et la reprise des ressources visionnaires et prophétiques de la transe comme voie de communication avec les esprits. Au Ghana, le terme même de "spiritual churches ", ou "spiritist ", correspond à une appellation locale indigène consacrée par la typologie du professeur C.G. Baeta (Prophetism in Ghana, 1962), et désigne des Églises prophétiques du même type apparues d'ailleurs simultanément dans les années 1920/1930, dans le prolongement de l'épopée harriste, et aujourd'hui marginalisées et stigmatisées par la vague du néo-pentecôtisme. L'absence de lien attesté d'emprunt ne peut faire oublier que les échanges réciproques entre les Églises noires américaines et les leaders des réveils des pays africains anglophones (Nigeria, Ghana), amorcés dès le XIX ${ }^{e}$ siècle par les returnees du Liberia et de la Sierra Leone, ont été fortement relancés dans le cadre des mouvements prophétiques du début du $\mathrm{xx}^{\mathrm{e}}$ siècle.

3 L'auteur nous avait déjà mis en appétit sur ce sujet à travers quelques articles qui sont ici repris dans une monographie de ces Églises affiliées, fondées pour la plupart dans le courant des années 1930, mais où domine, parmi bien d'autres dénominations, le cas de figure d'Israël Universal Spiritual Churches of Christ fondée en 1951 par l'archevêque E.J. Johnson. Cette monographie n'est cependant pas d'un genre très classique puisque toute l'approche se fait par touches successives (une série de "diapositives anthropologiques ", si l'on peut dire), en tournant autour de l'objet qui se dévoile par fragments ou recouvrements avec d'autres dynamiques religieuses (baptisme noir ou hoodoo d'origine haïtienne, chap.2) ou mouvements culturels plus connus (jazz et gospel, chap.3). Les récits d'initiation autobiographiques de Zora Neale Hurston, romancière et ethnologue, permettent judicieusement de témoigner, dans ce monde de la Louisiane des années 1920, d'une osmose entre les rituels du hoodoo louisianais, qui intègrent dans une matrice initiatique africaine des éléments de la symbolique catholique et de son culte des saints, et le monde des Églises spirituelles qui cherchent à se démarquer des Églises baptistes noires. Le terme spiritual (ou spiritualist qui l'a précédé) fait déjà doublement écho au mouvement du spiritisme et à ses medium, autant qu'à la tradition des negro spirituals, même si l'autre matrice d'inspiration est bien la spiritualité de l'Esprit saint héritée du courant baptiste. L'Esprit fait cependant bon ménage avec les esprits $\mathrm{du}$ monde du hoodoo pour les combattre ou les domestiquer, et le poids des ressources symboliques du catholicisme créole, avec ses autels, son encens, ses bougies, ses statues de saints, et ses costumes d'évêques, a 
fortement marqué l'esprit des fondateurs issus de l'élite catholique métisse de la Nouvelle-Orléans des années 1920.

4 Cet alliage singulier d'africanité initiatique, de ritualisme catholique, et de spiritualité baptiste et pentecôtiste, n'a rien d'une simple mosaïque d'emprunts, ni d'ailleurs non plus d'un mélange dit syncrétique. Ce syncrétisme est manifestement vécu par les leaders et les adeptes sur le mode d'un continuum culturel et religieux ignorant les incompatibilités ou les écarts (entre l'Esprit saint et les esprits, le fétichisme africain et la magie catholique, ou entre la musique du diable et celle du bon Dieu) que les autres (les Églises protestantes, catholiques, ou les mouvements identitaires afro-américains) s'évertuent à rappeler. Il est bien difficile de déceler dans le fondu enchaîné de pratiques chrétiennes et païennes quelque « affinité contradictoire » (p. 10), ou dans la cohabitation sereine des figures du culte quelque réinterprétation « dissimulée ». Il faut dire que la présence majeure de l'esprit indien de Black Hawk (p. 161-162), ce chef indien, héros de la résistance à la colonisation américaine, parmi les esprits protecteurs et intercesseurs de ces femmes pasteurs noires, plutôt accommodantes, porte à son comble l'hybridité de ce monde mêlé, plus ambigu et ambivalent qu'il n'y paraît.

La révélation du noyau spirituel de ces Églises, celle qui leur permet de se démarquer notamment du baptisme ou du pentecôtisme, est censée se trouver finalement dans une "théologie alternative", sous-jacente à la profession de foi commune de 1937, que l'ethnologue s'efforce de reconstituer, avec la complicité de quelque leader, sous le couvert symbolique de la "samaritaine noire ». Cette spécificité doctrinale ne manque pas de surprendre dans un monde où les fondateurs sont plutôt étrangers à la spéculation théologique et familiers d'une culture biblique à dominante orale, et où les disciples ignorent la plupart du temps le sens évangélique des pratiques initiatiques et thérapeutiques qui viennent répondre à leurs besoins pratiques. Comme l'avoue l'auteur du chapitre sur la «samaritaine noire»: «Nous ne disposons d'aucun texte herméneutique écrit par les fondateurs du mouvement spirituel sur ce sujet : nous en sommes réduits à des conjectures » (p. 88). L'exercice d'exégèse biblique auquel se livre l'ethnologue mis sur la piste de Jean, IV, par quelque informateur, évêque de son état, se révèle particulièrement inventif et même plausible, même si toutes les découvertes interprétatives restent formulées, en toute honnêteté, sur le mode du conditionnel, en l'absence de preuve indigène. "Dieu est Esprit », dit Jean, IV, qui fournit le dogme de base, sachant que la formule « God is $a$ spirit ", insiste sur l'idée que Dieu est une source de dons spirituels, et laisse la place heureusement à d'autres esprits (en soi ni bons ni mauvais) avec lesquels on peut entrer « directement » en communication. Le baptême dans l'esprit, qui prend le relais du baptême par immersion, inaugure cette alliance avec telle ou telle manifestation de l'esprit (de l'Esprit saint de Jésus à Black Hawk en passant par les saints catholiques) qui est avant tout source de divination, de prophétie et de guérison.

6 C'est ici qu'intervient, dans l'Évangile de Jean, la rencontre avec la femme samaritaine à qui Jésus demande de l'eau du puits. Cette scène emblématique témoigne d'un Jésus qui, à la différence de Jean, ne baptise pas lui-même par l'eau mais dispose d'un pouvoir prophétique : "Seigneur, je vois que tu es un prophète ». La samaritaine, cette figure féminine, élue entre toutes, incarnerait aux yeux des spirituels les promesses de salut offertes à un peuple paria (les Samaritains assimilables aux Africains descendants d'esclaves) et aux femmes à "plusieurs maris" des familles noires. L'hypothèse principale est que le symbole de la Samaritaine noire - dont la présence rituelle et 
liturgique est plutôt discrète, au regard des développements herméneutiques qui l'entourent - fournit la justification évangélique d'une vocation pastorale féminine. Et il est incontestable, comme le souligne l'auteur, que l'invention d'un pastorat féminin, non sans lien avec le rôle assumé par les femmes prêtresses du vaudou ou les mères de saints, est une vraie spécificité de genre de ces Églises, au regard de l'ensemble des autres Églises noires baptistes ou pentecôtistes.

7 Si les hommes finissent toujours dans ce monde par occuper les positions de hiérarques de la dénomination, évêques ou archevêques, ce sont bien les femmes, pasteurs et prophètes, qui sont les héroïnes de cette histoire d'Église. Ce n'est sans doute pas par hasard que le cycle rituel, qui constitue l'armature souterraine de l'ouvrage, commence par une cérémonie d'ordination rituelle de femmes sister promues au rang de mother, et se termine par le rituel funéraire de l'enterrement de Mother Mae. Dans la description d'une grande partie de ces séquences rituelles, comme dans l'évocation des rites d'initiation décrits par Hurston, l'auteur fait une large place à des textes datés des années 1930 ou 1940 dont la richesse descriptive dépasse ce que l'on peut sans doute observer aujourd'hui. Cette approche textuelle des séquences rituelles encourage, à défaut de l'observation des interactions en situation, une vraie passion de l'interprétation symbolique. Le recours à l'exégèse biblique des matériaux empruntés aux différents corpus tend à se développer, par libres associations ou conjectures, en l'absence du point de vue des acteurs (on pense entre autres au florilège de conjectures bibliques sur le sens de la symbolique du temple de Mère Seals, p. 60-62). L'enjeu est toujours de repérer dans ce "travail syncrétique » (p. 114), où s'élabore le sens d'une ordination féminine de la prêtrise, ce qui relève des éléments repris de telle ou telle tradition et ce qui décide du modèle, africain ou chrétien, qui ordonne in fine ces éléments, sachant qu'en définitive ce sont bien au moins deux «modèles» de référence, plutôt plastiques, qui cohabitent ou se télescopent dans le choix et surtout la réinterprétation des matériaux. Comment, dès lors, décider face à la description de ce rituel d'intronisation des "mères", "formellement similaire » aux cérémonies du mariage catholique, et plus précisément des vœux des novices qui deviennent les "épouses du Christ», de la pertinence du modèle chrétien plutôt que du modèle initiatique du vaudou inspiré du mariage mystique entre une initiée possédée et le loa ? L'autorité de la Mère Shannon qui, comme tous les leaders de la dénomination, nie toute complicité avec le hoodoo et déclare explicitement que les novices sont «mariées à Jésus » (p.97), ne peut empêcher à juste titre un anthropologue avisé des rites d'initiation des religions africaines de pratiquer pour sa part une lecture « en double». Et c'est bien ce double bind qui veut que la cérémonie soit chrétienne "et pourtant toujours païenne »-qu'elle est ce qu'elle est et qu'elle n'est pas ce qu'elle est - qui constitue le défi de ce type de posture ambivalente qui relevait pour Bastide d'un " principe de coupure ».

8 Cette parole en double n'est pas le produit d'une surinterprétation anthropologique, elle se donne à voir et à entendre dans le rituel lui-même puisque les futures mères, $\mathrm{y}$ compris la Mère Shannon, "parlent » à travers les transes qui les agitent toutes ou les paralysent, et disent manifestement autre chose que la formalité du rituel de passage catholique sur la nature de l'alliance mystique qui est en jeu. Le langage du corps n'est certainement pas univoque, mais il faut bien admettre que le corps agité et informé par l'esprit qui l'habite n'est pas seulement dans ce contexte une forme d'expression 
« émotionnelle » mais qu'il entre dans la négociation du sens de l'alliance mystique en décidant d'abord de ce qu'est un « esprit ».

9 Le couple conceptuel du «modèle » et de "l'élément », plus ou moins inspiré de la définition de la réinterprétation selon Herskovits, est un outil d'analyse un peu limité pour rendre compte de la dialectique de l'élément incorporé et du modèle réagencé qui engendre de telles formations de compromis. Que dire de la référence globale à une "structure commune» au schéma initiatique africain et au mariage mystique catholique ! L'intérêt de la structure devrait être au moins de permettre de comprendre la logique des transformations qui préside à ce genre de synthèses incompatibles, or la structure des rites de passage qui domine dans l'ouvrage toute la lecture des rites initiatiques et funéraires paraît là aussi un vêtement un peu large... à la fois pour fonder l'idée d'une "africanité initiatique» (p.40) et surtout pour rendre compte, à l'autre bout de la chaîne, des écarts et des enjeux spécifiques des funérailles catholiques et des rites funéraires africains. L'auteur apprécie beaucoup les tableaux croisés mais la matrice thanato-logique qu'il nous propose des rituels funéraires chrétiens ou autres (p. 187), sur la base de la conjonction/disjonction de l'âme et du corps, est, comme il le reconnait, un «squelette logique " qui manque un peu de chair... Comment rendre compte, par exemple, à partir de l'opposition âme/corps et des variantes qu'elle autorise, de l'irruption, au cœur de la cérémonie, de la transe du pasteur officiant qui aspergeait et encensait le cercueil, soudain terrassé par l'Esprit saint, et sans doute plus précisément par l'esprit de la défunte Mother Mae ? La "transe ", là encore, toujours au rendez-vous, témoigne, comme le note l'auteur, d'une sorte de "baptême inversé ", un baptême de l'esprit, s'entend, par lequel le charisme se transmet de la Mère défunte au Pasteur qui entend lui succéder. Cette circulation des esprits d'une personne à l'autre qui passe par les corps (du corps mort au corps vivant) bouscule manifestement le jeu abstrait de l'union et de la séparation de l'âme et du corps, et participe d'une conception très organique de la relation à l'Esprit, conçu comme un fluide qui envahit et pénètre le corps (p. 225).

10 Le rituel improvisé du balancement du corps de la défunte (à l'initiative des porteurs du cercueil et de la sœur de la défunte) offre un autre témoignage de la vie des esprits et de leur manipulation éventuelle en situation. Cette résurgence d'un fragment de mémoire familiale, au cœur d'un rituel public, aurait pu conduire, au-delà de l'effet de clin d'œil aux rites africains d'interrogation du cadavre, à prendre en compte très sérieusement les soupçons de sorcellerie qui planent sur tout enterrement, et entourent le sort des destins individuels. Les affaires quotidiennes de jalousie et de sorcellerie qui dominent la vie de l'Église et des fidèles attestent d'une autre forme de dédoublement de la personne que celle de l'esprit qui vous possède.

11 Le privilège accordé aux «rites de passage » conduit à mettre sous le boisseau la liturgie ordinaire qui fait la vie cultuelle des fidèles. Seule la présentation du film donne lieu à un exposé minimal de "l'organisation du service rituel» (p.225) et certaines séquences d'images permettent de prendre la mesure de cette atmosphère hybride de ritualisme catholique, de prédication protestante et de gospels évangéliques. Il faut attendre le chapitre 5 pour comprendre que la vie de l'Église, celle des pasteurs comme celle des "adeptes", se déroule, en fait, largement en dehors des temples et des cultes dominicaux qui ne rassemblent que très peu de fidèles (une trentaine au plus). Les lieux et les moments essentiels, ceux qui attestent du charisme des pasteurs et leur fournissent leurs ressources financières principales, ceux qui 
répondent aux attentes des adeptes et marquent leur rencontre avec le Christ ou d'autres esprits, ce sont les « consultations spirituelles » ordinaires qui se déroulent la plupart du temps en privé et non en public. Dans cet univers de la consultation, les attentes spirituelles se révèlent solidaires des malheurs qui affectent la population des quartiers noirs les plus pauvres de la Nouvelle-Orléans, et particulièrement les femmes: maladies et mort des proches, alcoolisme, drogue, etc. Les ressources prophétiques et divinatoires de la vision des esprits l'emportent sur les préoccupations théologiques et herméneutiques. Lorsque l'enquêteur se décide à donner la parole aux "pratiquants spirituels » de base (une trentaine ont été interviewés), et même aux leaders, s'il apparaît clairement que la « rencontre » avec l'Esprit (ou un esprit) est liée à «l'affliction» (Turner), et la conversion à une expérience de guérison miraculeuse qui passe entre autres par l'épuration du corps et l'exorcisme des forces du mal. Il est significatif que les séances de "développement spirituel» font une large place à l'identification des "mauvais sorts", et supposent de la part des spiritual doctors d'assumer parfois « le sale boulot » des contre-sorciers. Les pratiques de "délivrance » des Églises pentecôtistes et des communautés charismatiques américaines (voir T.J. Csordas) ne sont pas loin, mais l'ancrage des affaires de sorcellerie dans le registre des conflits familiaux et matrimoniaux et le recours aux médiations "fétichistes » (eau bénite, bougies, huile sainte) et aux protections « magiques » (bains, lavements et usage des plantes) évoquent plus directement l'univers des remèdes et « travaux » des Églises prophétiques africaines. La prégnance de cette culture visionnaire et de ces pratiques d'exorcisme est sans doute le plus fort témoignage d'une " africanité » qui interpelle et bouscule l'esprit de l'éthique protestante.

On ne peut que souhaiter, de ce point de vue, qu'un travail ou un développement plus approfondi soit accordé à cette culture visionnaire des consultations spirituelles. Comment se dessinent parmi les " pratiquants spirituels » les vocations de prophètes et de visionnaires, et celles-ci sont-elles consacrées au sein de la hiérarchie par des statuts particuliers, institués, sachant qu'on peut apparemment atteindre une position de hiérarque, surtout si on est un homme, sans disposer de ces charismes? Il est beaucoup question de séminaires de formation et de développement spirituel, et le suivi de ces derniers pourrait éclairer les techniques du corps ou les techniques de soi qui entretiennent les charismes des prophètes. Enfin, le film permet d'entrevoir une consultation spirituelle impromptue, à l'initiative de la prophétesse, à la limite entre l'interaction privée et la scène publique, qui n'hésite pas à orienter le soupçon de sorcellerie sur un membre de la famille également membre de l'Église (ici le conjoint de la consultante qui, de plus, est "évêque »). On aurait aimé savoir comment de tels diagnostics de sorcellerie, et les formes, même dissimulées, d'accusation, sont gérés au sein de cette communauté chrétienne.

Pour conclure, on aura compris que l'ouvrage s'accompagne d'un film au titre évocateur Roll With the Spirit réalisé par l'auteur à l'occasion d'une convention qui s'est réunie à Chicago en 2002. Le film, comme l'ouvrage, confirme l'importance de la culture de la transe dans les manifestations de cette religiosité festive, même si, comme dans toutes les traditions, le terme de «transe » tel que nous le comprenons n'est jamais adéquat aussi bien au regard de l'expérience vécue et de sa catégorisation religieuse. "Tomber en esprit » suppose néanmoins, chez les païens comme chez les chrétiens, tout un dispositif rituel faisant appel à des rythmes musicaux, des incantations orales, une codification des conduites et des techniques du corps, et même des agents de surveillance et de bienveillance (sans parler de la caméra ou du cameraman qui font 
souvent partie de la mise en scène). La séquence finale du film donne à voir et à entendre une séance privée de relations à plaisanterie, aux lendemains du culte et après la retombée de ses moments de fortes émotions, où une actrice imite les gestes et expressions des uns et des autres en provoquant l'hilarité générale. Cette séquence remarquable contribue incontestablement à illustrer la « réflexivité » d'une expérience à la fois spirituelle et corporelle, domestiquée et stylisée, que l'on assimile à tort à quelque liturgie émotionnelle témoignant de l'expression immédiate des manifestations de l'esprit. La "réflexivité » est un objet d'attention important dans l'anthropologie actuelle des formes de l'action rituelle. Les possédés, comme les convertis, ne participent pas de la plénitude d'un état qui confine à l'oubli de soi, mais la réflexivité des états de conscience ou de croyance des sujets prend sa vraie dimension autant dans la relation aux autres (ici la transe des autres, p. 230) que dans la relation de soi à soi (ou à l'autre soi qui est «mon» esprit), une question pendant longtemps complètement occultée dans l'expérience de la possession. 